\title{
Electrical and Optical Properties of Zinc Oxide Thin Films Deposited Using Atomic Layer Deposition
}

\author{
Jeong-Eun Kim, Seung-Muk Bae, Heesun Yang, and Jin-Ha Hwang ${ }^{\dagger}$ \\ Department of Materials Science and Engineering, Hongik Univeristy, Seoul 121-791, Korea \\ (Received July 1, 2010; Revised July 18, 2010; Accepted July 19, 2010)
}

\begin{abstract}
Zinc oxide $(\mathrm{ZnO})$ thin films were deposited using atomic layer deposition. The electrical and optical properties were characterized using Hall measurements, spectroscopic ellipsometry and UV-visible spectrophotometry. The electronic concentration and the mobility were found to be critically dependent on the deposition temperature, exhibiting increased resistivity and reduced electronic mobility at low temperature. The corresponding optical properties were measured as a function of photon energy ranging from 1.5 to $5.0 \mathrm{eV}$. The simulated extinction coefficients allowed the determination of optical band gaps, i.e., ranging from 3.36 to $3.41 \mathrm{eV}$. The electronic carrier concentration appears to be related to the reduction in the corresponding band gap in $\mathrm{ZnO}$ thin films.
\end{abstract}

Key words : Zinc oxide, Atomic layer deposition, Resistivity, Spectroscopic ellipsometry, Band gaps

\section{Introduction}

$\mathrm{Z}$ inc oxide $(\mathrm{ZnO})$ has been gaining extensive attention as a key-element material in transparent optoelectronics in addition to increasing the diversity of spintronic and electronic devices. The representative applications of $\mathrm{ZnO}$-based materials incorporate transparent conducting electrodes and transparent channel materials; examples of the former include Al-doped $\mathrm{ZnO}$ (AZO) and Ga-doped $\mathrm{ZnO}$ (GZO); examples of the latter include In-Zn-Ga-O, Hf-ZnGa-O, Zn-Sn-O, etc. ${ }^{1-8)}$ The field of transparent conducting oxides requires the replacement of expensive indium-based conducting electrodes due to the scarcity of indium metals. Possible alternatives can be provided through the introduction of $\mathrm{ZnO}$-based materials owing to the cheaper and nontoxic features of the elemental Zn source. Usually, transparent conducting oxides function as electrodes for photovoltaic cells, flat-panel displays, etc, enabled by high transmittance in the visible light regime and their high conductivity. In addition, the semiconducting feature of $\mathrm{ZnO}$-based materials allows the fabrication of transparent oxide thin film transistors functioning as switching circuits in activematrix flat-panel displays, such as organic light-emitting diodes, liquid-crystal displays, etc. ${ }^{4-8)}$ In spite of the industrial significance of $\mathrm{ZnO}$ materials, their inherent electrical and optical features remain unresolved in terms of electrical transport and optical characteristics.

Atomic layer deposition usually provides unprecedented benefits such as excellent thickness uniformity, conformal

${ }^{\dagger}$ Corresponding author: Jin-Ha Hwang

E-mail : jhwang@hongik.ac.kr

Tel : +82-2-320-3069 Fax : +82-2-333-0127 deposition, and high level of step coverage, despite its slow process, which stems from alternating supplies of source materials in connection with purging steps. ${ }^{9,10)}$ Recently, low temperature processing capability has been recognized as an advantage for the fabrication of amorphous oxide semiconductor thin film transistors.

The present work is aimed at the electrical and optical characterization of $\mathrm{ZnO}$ thin films deposited using self-limiting atomic layer deposition at low temperature. The electrical features were examined through Hall measurements and the optical characteristics were analyzed using spectroscopic Ellipsometry and UV-visible spectrophotometry. The implications of $\mathrm{ZnO}$ deposited through atomic layer deposition are discussed towards the goal of achieving sophisticated control in semiconducting behavior.

\section{Experimental Procedure}

$\mathrm{ZnO}$ thin films were deposited using atomic layer deposition in which diethylzinc (DEZ) and water were employed as sources for $\mathrm{Zn}$ and oxygen, respectively. $\mathrm{ZnO}$ thin films were deposited onto high-insulating glass substrates (Corning 1737) after appropriate cleansing procedures. Alternating sources of DEZ and water were introduced to the deposition chamber in-between with alternating purging processes using argon gas; the deposition temperature was controlled between 100 and $250^{\circ} \mathrm{C}$.

The electrical characterization was made under ambient conditions using a Hall measurement unit (Bio-Rads, USA). The optical measurement was performed using spectroscopic ellipsometry (Horiba, Japan). The $(\Delta, \Phi)$ spectra values were measured as a function of photon energy ranging from 1.5 to $5.0 \mathrm{eV}$, for which $\Phi$ and $\Delta$ denote the change in amplitude and the phase shift in p- and s-polarized light, 


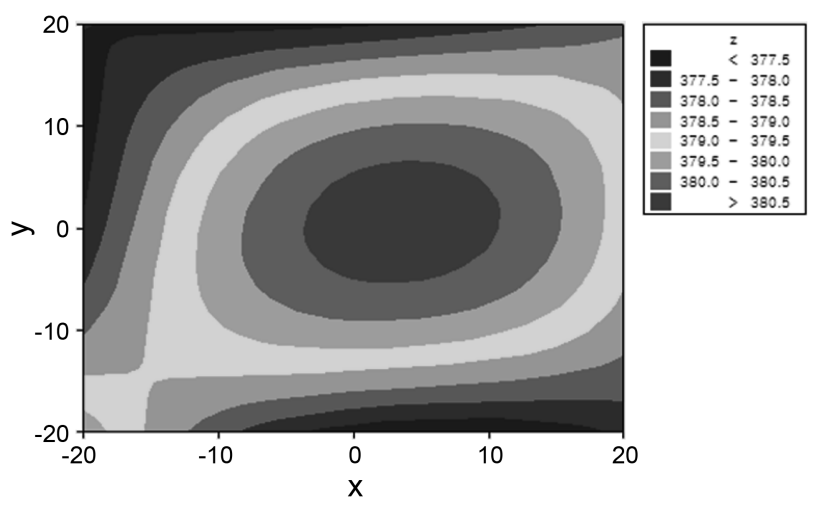

Fig. 1. Two-dimensional thickness contour obtained in the $\mathrm{ZnO}$ thin film deposited at $100^{\circ} \mathrm{C}$ using atomic layer deposition.

respectively, before and after the incident light is transmitted onto the optical system composed of $\mathrm{ZnO}$ thin films and glass substrates. Optical transmittance was measured between 300 and $900 \mathrm{~nm}$, using a UV-visible spectrophotometry (Shimadzu, Japan) in which the contribution of the glass substrates was corrected in order to measure the inherent contribution to transmittance, originating from the $\mathrm{ZnO}$ thin films deposited through atomic layer deposition.

\section{Results and Discussion}

The atomic layer deposition of $\mathrm{ZnO}$ thin films allows high thickness uniformity as shown in Fig. 1. The thickness uniformity was calculated at $0.46 \%$ based on the maximum and minimum thickness obtained from the $40 \mathrm{~mm} \times 40 \mathrm{~mm}$ glass substrates; the thickness profile seems radially isotropic with respect to the center of the deposition chamber. From the thickness information, the deposition rate was calculated to be approximately $0.12 \mathrm{~nm} /$ cycle. The band gap of the bulk zinc oxide materials is known to be approximately $3.3 \mathrm{eV}{ }^{11)}$ The high band gap leads to a superior transparency in the visible light region. The current $\mathrm{ZnO}$ thin film exhibits the high transmittance, higher $80 \%$ above $400 \mathrm{~nm}$, characteristic of the transparent oxides. The optical transmittance was measured at between 92.5 and $94.0 \%$ at a wavelength of $600 \mathrm{~nm}$. Fig. 2 indicates that the transmittance seems to be nearly independent of the deposition temperatures between 100 and $250^{\circ} \mathrm{C}$.

Spectroscopic ellipsometry allows the numerical determination of optical constants, i.e., refractive index and extinction coefficient in addition to the thickness determination. Fig. 3(a) shows the experimental spectroscopic ellipsometry ( $\Phi$ and $\Delta$ ) spectra obtained from the $\mathrm{ZnO}$ thin films, where $\Phi$ denotes the change of amplitude and $\Delta$ the change in phase shift before and after the interaction of the incident light with the optical structure (i.e., the measuring system of the $\mathrm{ZnO}$ (films)/glass structure). Based on the optical structure shown in Fig. 3(b), ellipsometry modeling was performed in order to statistically minimize the deviation

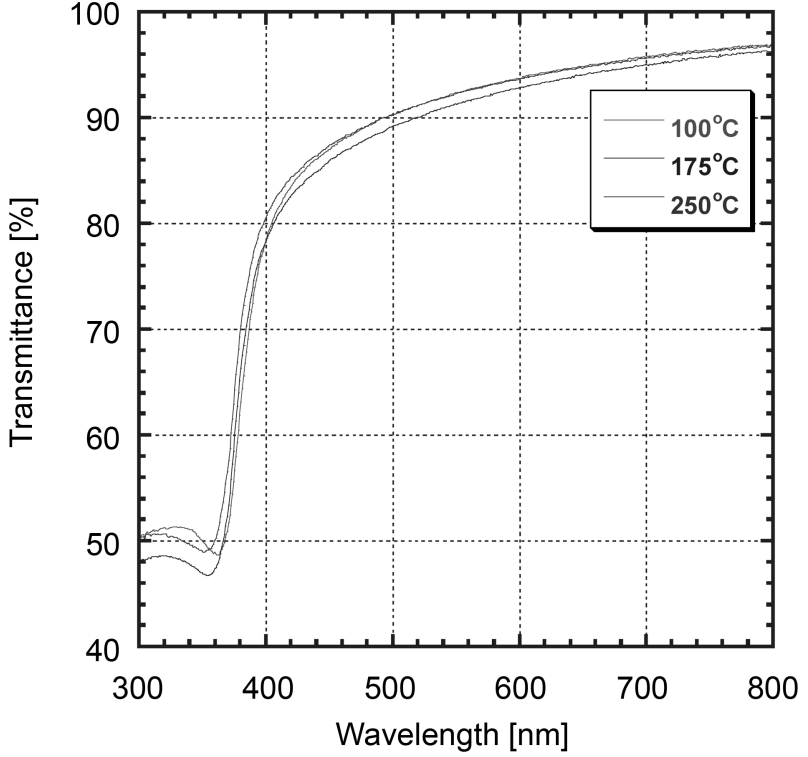

Fig. 2. Transmittance of $\mathrm{ZnO}$ thin films deposited through atomic layer deposition measured using UV-visible spectrophotometry.

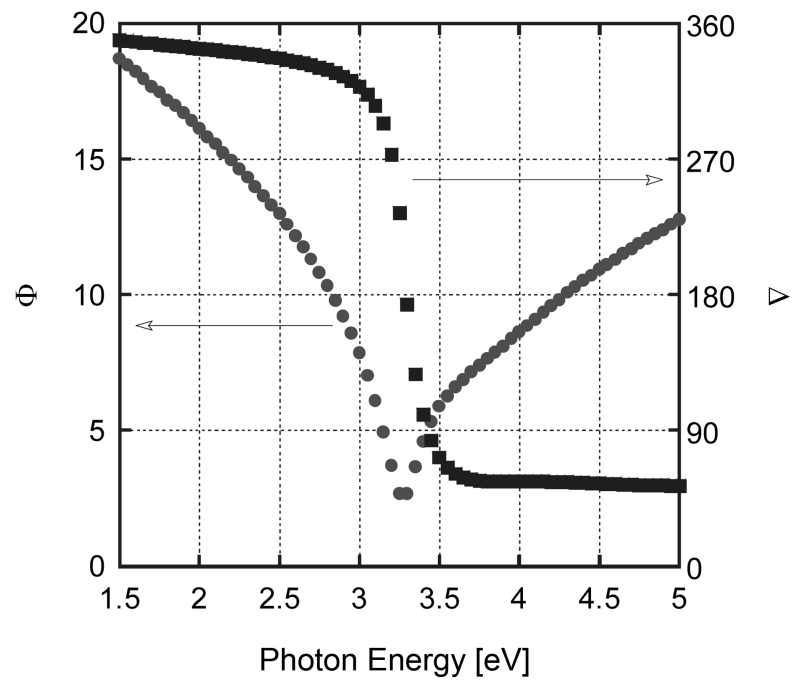

(a)

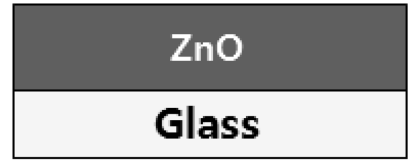

(b)

Fig. 3. (a) $(\Phi, \Delta)$ spectra obtained from the $\mathrm{ZnO}$ thin films deposited at $100^{\circ} \mathrm{C}$ and (b) optical structure employed in spectroscopic ellipsometry analyses.

between the experimental and the fitted $\Phi$ and $\Delta$ spectra. In this work, the ellipsometry modeling adopts a new amorphous model as a dielectric model for $\mathrm{ZnO}$ thin films. The detailed equations are described with respect to the refractive index $n(w)$ and the extinction coefficient $k(w)$ as follows: 


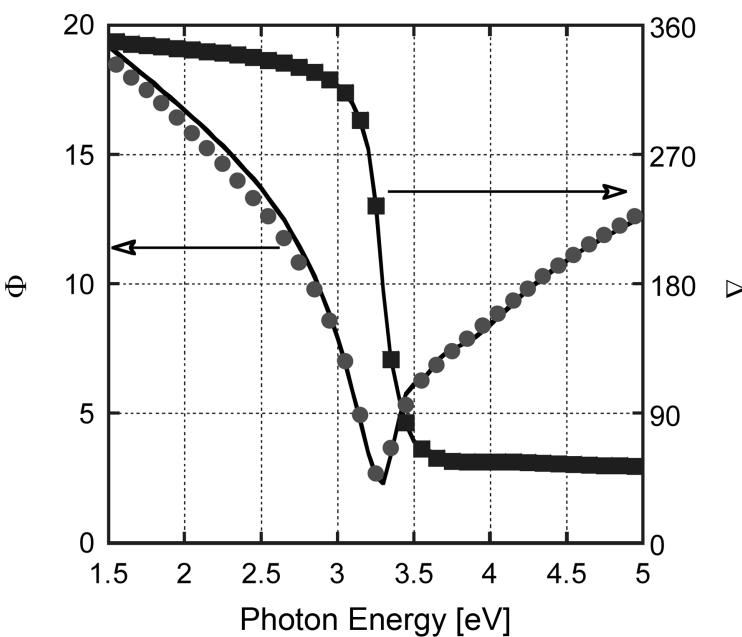

(a)

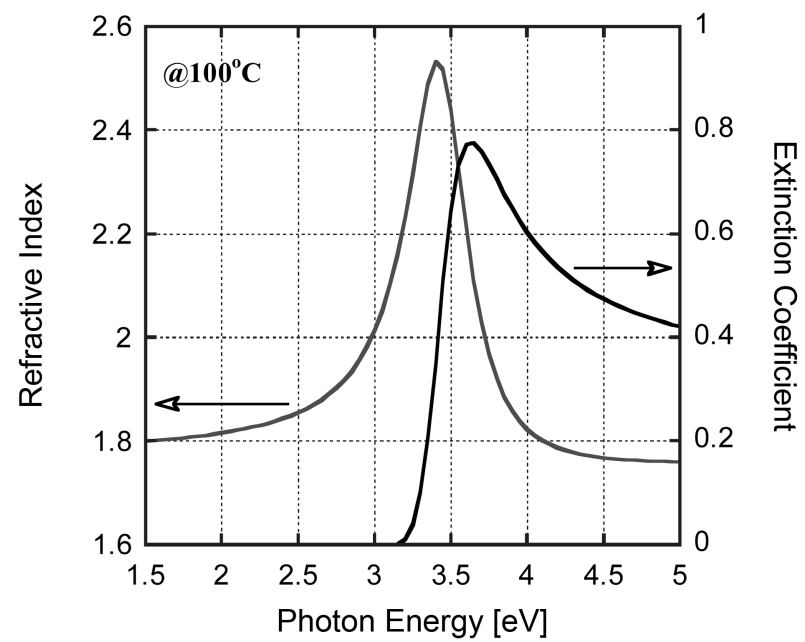

(b)

Fig. 4. (a) $(\Delta, \Phi)$ spectra fitted using a new amorphous model in which the solid circles and squares denote the raw spectra data points and the solid lines indicate the fitted plots and (b) the calculated optical constants of refractive index and extinction coefficient.

$$
\begin{aligned}
& n(\omega)=n_{\infty}+\frac{B \cdot\left(\omega-\omega_{j}\right)}{\left(\omega-\omega_{j}\right)^{2}+\Gamma_{j}^{2}}, \\
& k(\omega)=\left\{\begin{array}{cl}
\frac{f \cdot\left(\omega-\omega_{g}\right)^{2}}{\left(\omega-\omega_{j}\right)^{2}+\Gamma_{j}^{2}}, & \omega>\omega_{g} \\
0, & \omega \leq \omega_{g}
\end{array}\right.
\end{aligned}
$$

where

$$
\begin{aligned}
& B=\frac{J_{j}}{\Gamma_{j}} \cdot\left(\Gamma_{j}^{2}-\left(\omega_{j}-\omega_{g}\right)^{2}\right), \\
& C=2 \cdot f_{j} \cdot \Gamma_{j} \cdot\left(\omega_{j}-\omega_{g}\right)
\end{aligned}
$$

Based on the new amorphous model, the experimental

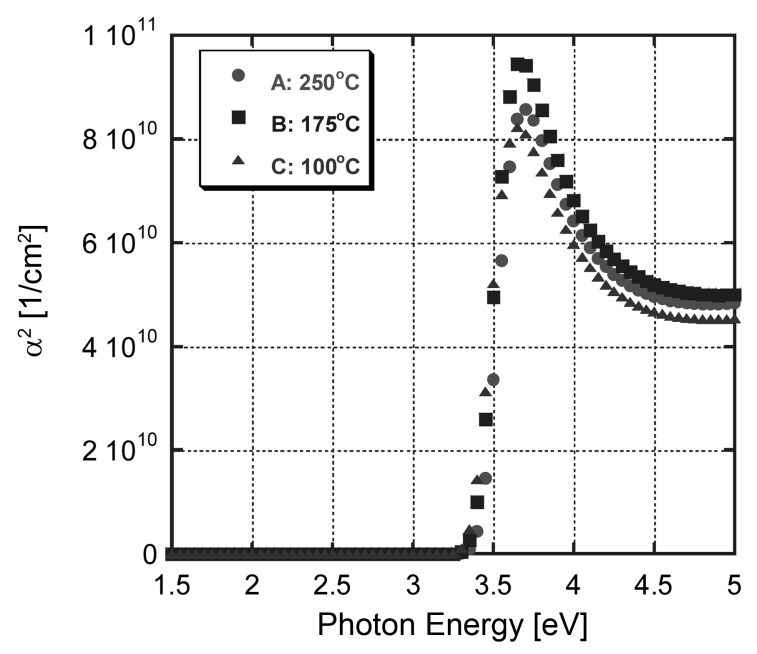

(a)

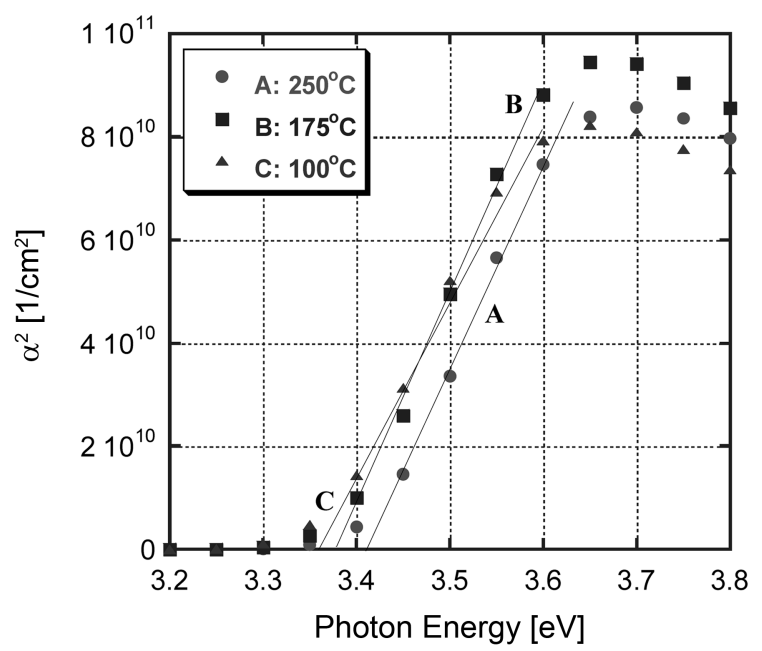

(b)

Fig. 5. (a) Calculated absorption coefficient and (b) extrapolation of band gaps near the optical band gaps.

spectra were analyzed and the resultant fitting information is shown in Fig. 4(a). The numerical fitting is in excellent agreement with the experimental $(\Phi, \Delta)$ spectra. The optimized optical parameters allow the determination of the basic optical constants, i.e., the refractive index and the extinction coefficients of the following equation in the corresponding complex refractive index, $N(w)$,

$$
N(w)=n(w)-i k(w)
$$

The calculated optical refractive index and the extinction coefficient are shown in Fig. 4(b). The refractive index exhibits its highest value near $3.4 \mathrm{eV}$ and the absorption becomes noticeable above $3.2 \mathrm{eV}$. In particular, the extinction coefficient is employed in order to calculate the absorption coefficient, $\alpha\left(E_{n}\right)$.

$\alpha\left(E_{n}\right)=4 \pi k(w) / \lambda$

Since $\mathrm{ZnO}$ materials are known to be direct semiconduc- 
Table 1. Summarized Electrical Information Obtained in $\mathrm{ZnO}$ Thin Films Prepared Through Atomic Layer Deposition

\begin{tabular}{ccccc}
\hline $\begin{array}{c}\text { Deposition } \\
\text { Temp. }\end{array}$ & $\begin{array}{c}\text { Sheet } \\
\text { Resistance } \\
(\Omega / \mathrm{sq})\end{array}$ & $\begin{array}{c}\text { Bulk } \\
\text { Resistivity } \\
(\Omega \cdot \mathrm{cm})\end{array}$ & $\begin{array}{c}\text { Mobility } \\
\left(\mathrm{cm}^{2} / \mathrm{V} \cdot \mathrm{s}\right)\end{array}$ & $\begin{array}{c}\text { Charge Carrier } \\
\text { Concentration } \\
\left(\# / \mathrm{cm}^{3}\right)\end{array}$ \\
\hline $250^{\circ} \mathrm{C}$ & $1.82 \times 10^{3}$ & 0.00636 & 8.6 & $1.14 \times 10^{20}$ \\
\hline $175^{\circ} \mathrm{C}$ & $1.67 \times 10^{3}$ & 0.00583 & 11.2 & $9.52 \times 10^{19}$ \\
\hline $100^{\circ} \mathrm{C}$ & $2.13 \times 10^{5}$ & 0.746 & 0.451 & $1.86 \times 10^{19}$ \\
\hline
\end{tabular}

tors, the following equation describes the relationship between the absorption coefficient and the photon energy.

$$
\alpha\left(E_{n}\right)=A\left(E_{n}-E_{g}\right)^{1 / 2}
$$

where $E_{n}$ is the photon energy ranging from 1.5 to $5.0 \mathrm{eV}$ and $E_{g}$ is the band gap of $\mathrm{ZnO}$ thin film. ${ }^{12)}$ Fig. 5 shows the variation of the absorption coefficient of $\mathrm{ZnO}$ thin films as a function of photon energy. As noticed in Fig. 5(a), a significant increase in the absorption coefficient near $3.3 \mathrm{eV}$ is found. Detailed calculation leads to the determination that the band gaps of $\mathrm{ZnO}$ thin films range from 3.36 to $3.41 \mathrm{eV}$, due to the modified relations between $\alpha\left(E_{n}\right)^{2}$ and the photon energy $\left(E_{n}\right)$, as demonstrated in Fig. 5(b), indicating that the higher the deposition temperature, the larger the band gap of the $\mathrm{ZnO}$ thin film.

The electrical features of $\mathrm{ZnO}$ thin films were monitored through Hall measurements. The detailed information is summarized in Table 1. With decreasing deposition temperature, the resistivity increased from $6.36 \times 10^{-3}$ to $7.46 \times 10^{-1} \Omega \cdot \mathrm{cm}$. However, the mobility decreased from 8.6 to $0.451 \mathrm{~cm}^{2} / \mathrm{V} \cdot \mathrm{s}$ and the charge carrier concentration decreased from $1.14 \times 10^{20}$ to $1.86 \times 10^{19} / \mathrm{cm}^{3}$. The measured resistivity indicates that the corresponding electrical behavior is attributable to the oxide-based semiconductors. The electrical information is believed to be associated with the defect-chemical responses in the $\mathrm{ZnO}$ in which zinc interstitials play a significant role in the charge transport phenomena. The higher deposition temperature provides a higher formation of zinc interstitials along with predominantly electronic conductions.

$\mathrm{ZnO}$ thin films were deposited with high uniformity in thickness which is controlled layer-by-layer, in a way characteristic of atomic layer deposition. The high-precision spectroscopic ellipsometry analysis allowed the numerical calculation of optical band gaps in addition to the calculation of optical constants. Furthermore, the electrical Hall measurements revealed that the charge carrier concentration and mobility are sensitive to deposition temperature. Atomic layer deposition of $\mathrm{ZnO}$ thin films can be exploited aiming at the artificial control of charge concentration and mobility in transparent oxide thin film transistors.

\section{Conclusions}

Electrical and optical characterization confirmed the presence of the semiconducting behavior in $\mathrm{ZnO}$ thin films deposited on glass substrates through atomic layer deposition. The optical constants, i.e., the refractive index and the extinction coefficient, are estimated through optical simulation based on spectroscopic ellipsometry. The direct band gap structure is related to the absorption coefficients in the $\mathrm{ZnO}$ thin films and the optical band gaps were calculated to be approximately 3.36 to $3.41 \mathrm{eV}$. Despite the large band gap in $\mathrm{ZnO}$ thin films, the charge transport is still semiconducting, probably due to the presence of zinc interstitials. The electrical resistivity increases with decrease of the growth temperature along with the simultaneous decrease in electronic concentration.

\section{Acknowledgments}

This work was supported by the Korea Research Foundation Grant funded by the Korean Government (MOEHRD, Basic Research Promotion Fund) (KRF-2007-314-D00162).

\section{REFERENCES}

1. J.-H. Park, K.-J. Ahn, K.-I. Park, S.-I. Na, and H.-K. Kim, “An Al-Doped ZnO Electrode Grown by Highly Efficient Cylindrical Rotating Magnetron Sputtering for Low Cost Organic Photovoltaics," J. Phys. D: Appl. Phys., 43115101 1-6 (2010).

2. S.-M. Park, T. Ikegami, and K. Ebihara, "Effects of Substrate Temperature on the Properties of Ga-doped $\mathrm{ZnO}$ by Pulsed Laser Deposition," Thin Solid Films, 513 90-4 (2006).

3. H.Kim, J.S.Horwitz, S.B.Qadri, and D.B.Chrisey, "Epitaxial Growth of Al-doped ZnO Thin Films Grown by Pulsed Laser Deposition," Thin Solid Films, 420-21 107-11 (2002).

4. R. L. Hoffman, B. J. Norris, and J. F. Wager, "ZnO-based Transparent Thin-Film Transistors," Appl. Phys. Lett., 82 [53] 733-35 (2005).

5. K. Nomura, H. Ohta, K. Ueda, T. Kamiya, M. Hirano, and H. Hosono, "Thin-Film Transistor Fabricated in SingleCrystalline Transparent Oxide Semiconductor," Science, 300 1269-72 (2003).

6. E. Chong, K. C. Jo, and S. Y. Lee, "High Stability of Amorphous Hafnium-Indium-Zinc-Oxide Thin Film Transistor," Appl. Phys. Lett., 96152102 1-3 (2010).

7. M. Ito, M. Kon, C. Miyazaki, N. Ikeda, M. Ishizaki, R. Matsubara, Y. Ugajin, and N. Sekine, "Amorphous Oxide TFT and Their Applications in Electrophoretic Displays," Physica Status Solidi (a), 205 [8] 1885-94 (2008).

8. Y. Ohya, T. Niwa, T. Ban, and Y. Takahashi, "Thin Film Transistor of ZnO Fabricated by Chemical Solution Deposition," Jpn. J. Appl. Phys., 40 297-98 (2001).

9. M. Leskela and M. Ritala, "Atomic Layer Deposition (ALD): from Precursors to Thin Film Structures," Thin Solid Films, 409 138-46 (2002).

10. M. Ritala, M. Leskelä, J.-P. Dekker, C. Mutsaers, P. J. Soininen, and J. Skarp, "Perfectly Conformal TiN and $\mathrm{Al}_{2} \mathrm{O}_{3}$ Films Deposited by Atomic Layer Deposition," Chem. Vap. Dep., 5 [1] 7-9 (1999).

11. V. Srikant and D. R. Clarke, "On the Optical Band Gap of Zinc Oxide," J. Appl. Phys., 83 [10] 5447-51 (1998).

12. H. Fujiwara, Data Analysis Examples in Spectroscopic Ellipsometry: Principles and Applications, pp. 249-310, John Wiley \& Sons. Ltd. Chichester, England 2007. 